\title{
Utility of fibrin sealants and the road ahead in plastic surgery: a scoping review
}

\section{Abstract \\ Objective:}

The objective of this scoping review is to examine the extent of literature on the utility and safety of various types of fibrin sealants in plastic surgery. Considerations such as consent, and cost profile will also be reviewed and identified as future research opportunities.

\section{Introduction:}

Since the approval of fibrin sealants in Europe in the 1970s, they have found their niche in various types of surgical operations, and their utility has led to a rise in popularity. However, the clinical applications of fibrin sealants are not fully synthesized, and important ethical and financial considerations are rarely discussed.

\section{Inclusion criteria:}

Studies reporting the application of commercially available fibrin sealants in plastic surgery procedures on humans of all age groups were included in this review. Additionally, published abstracts and all opinion pieces including commentaries and narrative reviews were exempt.

\section{Methods:}

The key information sources that will be searched are the following: Ovid MEDLINE, Cochrane Central Register of Controlled Trials (CENTRAL), Cochrane Database of Systematic Reviews, Embase, Web of Science Core Collection, and LILACS and grey literature sources. There are no limits placed on the searches. Each publication will be screened by two authors, and any conflict in the selection decision will be resolved by a third author to limit bias.

Keywords: Fibrin sealant; surgical adhesives; plastic and reconstructive surgery; scoping review; hemostatic agent

\section{Abstract word count: 205}

Total manuscript word count: 1011 


\section{Introduction}

Since the approval of fibrin sealants (FS) in Europe in the 1970s, FS have made their way into various types of surgical operations, such as cardiothoracic, orthopedic, neuro, and plastic surgery as hemostatic and tissue binding agents. (1) After approval in North America in 1998 their off-label utility has expanded to tissue engineering and drug-delivery, leading to a rise in their popularity. (1) With continual scientific and medical advancements, there has been an increased demand for more novel and efficient surgical techniques, further encouraging researchers and surgeons to push the boundaries on the utility of FS. Plastic and reconstructive surgical procedures contain an added element of aesthetic outcomes that can further add to the complexity of their utility in various surgical procedures.

Nearly 200 papers discussing FS are published per year, expanding the body of literature on the use of FS in the past two decades. (2) The literature primarily details the utility and safety profiles of the various FS available; however, the clinical application is not fully synthesized, and important ethical and financial considerations associated with the use of FS are rarely discussed. A comprehensive review that can aid clinical decision-making of medical professionals regarding the utility of FS in plastic surgery has not been published yet.

The objective of this scoping review is to explore the breadth of literature on the utility of various types of FS, in addition to summarizing indications and contraindications, side effects, and adverse events associated with their use in plastic surgery. Important considerations such as consent, and cost will also be reviewed and identified as future research opportunities. With this review, we aim to create a guide for surgeons and residents to further expand their knowledge on the utility of FS in plastic reconstructive surgical procedures.

A preliminary search of PROSPERO, MEDLINE and the Cochrane Database of Systematic Reviews was conducted and no current or underway scoping reviews or systematic reviews on the topic were identified.

\section{Review question(s)}

- What alternatives are available to human-derived fibrin sealants for patients who do not consent to the use of conventional fibrin glue formulations?

- Does consent need to be considered for the use of human-derived fibrin sealants?

- Does consent need to be aligned with consent for other blood-bank products for human-derived products?

- What are the current fibrin glue delivery systems used in plastic surgery procedures?

- What is the breadth of applications of fibrin sealants in plastic surgery?

- What are the off-label uses (drug delivery or tissue engineering medium) of fibrin sealants in plastic surgery?

- What side effects or adverse events are recognized with the use of various fibrin sealants in plastic 
surgery

- What contraindications should be considered before the use of fibrin sealants?

- What are the financial implications of fibrin sealants in plastic surgery?

\section{Inclusion criteria}

This review will consider studies that include human participants of any age, ethnicity, gender or from any geographical area. In addition, studies that explore the use of fibrin sealants as an intervention in any plastic or reconstructive surgical operation will be included. This scoping review will consider quantitative, qualitative, and mixed methods study designs for inclusion. In addition, systematic reviews and case reports will be examined for inclusion in the proposed scoping review. Commentaries, narrative, opinion pieces and abstracts will be excluded.

\section{Methods}

The proposed scoping review will be conducted in accordance with the JBI methodology for scoping reviews. (3)

\section{Search strategy}

The search strategy will aim to locate both published and unpublished primary studies, and reviews. An initial limited search of MEDLINE (Ovid) will be undertaken to identify articles on the topic. The text words contained in the titles and abstracts of relevant articles, and the index terms used to describe the articles will be used to develop a full search strategy. The search strategy, including all identified keywords and index terms, will be adapted for each included information source.

The databases to be searched include MEDLINE (Ovid), Cochrane Central Register of Controlled Trials (CENTRAL; Ovid), Cochrane Database of Systematic Reviews (Ovid), Embase (Ovid), Web of Science Core Collection, LILACS.Sources of unpublished studies and gray literature to be searched include Google Scholar, Dissertations \& Theses Global (ProQuest), OAlster (limited to theses), WHO International Clinical Trials Registry Platform, PapersFirst (WorldCat) and Proceedings (WorldCar). No limits were placed on the search.

Articles published in any language will be considered. Articles published from 1972 to the present will be included as fibrin sealants were only approved in Europe in 1972 and 1998 in North America. (1) 


\section{Study/Source of evidence selection}

References were uploaded into Covidence, and duplicates removed. Following a pilot test, titles and abstracts will then be screened by two independent reviewers for assessment against the inclusion criteria for the review. The full text of selected citations will be assessed in detail against the inclusion criteria by two independent reviewers. Reasons for exclusion of full-text papers that do not meet the inclusion criteria will be recorded and reported in the scoping review. Any disagreements that arise between the reviewers at each stage of the selection process will be resolved through discussion or with a third reviewer. The results of the search will be reported in full in the final scoping review and presented in a Preferred Reporting Items for Systematic Reviews and Meta-analyses for Scoping Reviews (PRISMA-ScR) flow diagram. (4)

\section{Data extraction}

Data will be extracted from papers included in the scoping review by three independent reviewers using Covidence. The data extracted will include specific details about the utility of various types of FS, indications and contraindications, side effects, and adverse events associated with their use in plastic surgery on humans of all ages. (5) A draft extraction tool is provided. The draft data extraction tool will be modified and revised as necessary during the process of extracting data from each included paper. Modifications will be detailed in the full scoping review. Any disagreements that arise between the reviewers will be resolved through discussion or with a third reviewer. Authors of papers will be contacted to request missing or additional data, where required.

\section{Acknowledgments}

There is no acknowledgement to declare.

\section{Funding}

Grant funding for this project was provided by the Office of Pediatric and Surgical Education and Innovation (OPSEI) from the University of British Columbia (UBC).

\section{Conflicts of interest}

The authors declare no conflict of interest.

\section{References}

(1) Radosevich M. Fibrin sealant: scientific rationale, production methods, properties, and current clinical use. Vox sanguinis 1997;72(3):133-143. 
(2) Spotnitz WD. Fibrin Sealant: The Only Approved Hemostat, Sealant, and Adhesive-a Laboratory and Clinical Perspective. ISRN surgery 2014;2014:1-28.

(3) Peters MDJ, Godfrey C, McInerney P, Munn Z, Tricco AC, Khalil, H. Chapter 11: Scoping Reviews (2020 version). In: Aromataris E, Munn Z (Editors). JBI Manual for Evidence Synthesis, JBI, 2020. Available from: https://synthesismanual.jbi.global.

(4) Tricco AC, Lillie E, Zarin W, O'Brien KK, Colquhoun H, Levac D, et al. PRISMA extension for scoping reviews (PRISMA-ScR): checklist and explanation. The PRISMA-ScR Statement. Ann Intern Med. 2018;169(7):467-73

(5) Babineau J. Product Review: Covidence (Systematic Review Software) [Internet]. Journal of the Canadian Health Libraries Association / Journal de l'Association des bibliothèques de la santé du Canada. [cited 2021Jun22]. 


\section{Appendix I: Search strategy}

MEDLINE (Ovid). Search conducted in March 2021.

\begin{tabular}{|c|c|c|}
\hline Search & Query & $\begin{array}{l}\text { Records } \\
\text { retrieved }\end{array}$ \\
\hline \#1 & Fibrin Tissue Adhesive/ & 4842 \\
\hline \#2 & Fibrin Tissue Adhesive.nm. & 4842 \\
\hline \#3 & ((fibrin or tissue) adj2 (glue or adhesive? or sealant? or spray)).tw. & 8475 \\
\hline \#4 & ((fibrin or tissue) and (glue or adhesive? or sealant? or spray)).kf. & 933 \\
\hline \#5 & (Artiss or Tisseel or Evicel or TachoSil or Evarrest or Raplixa).tw,kf. & 726 \\
\hline \#6 & or/1-5 & 9928 \\
\hline \#7 & Surgery, Plastic/ & 26737 \\
\hline \#8 & Reconstructive Surgical Procedures/ & 52370 \\
\hline \#9 & (plastic or reconstructive).tw,kf,nw. & 213515 \\
\hline \#10 & reconstruction.tw,kf. & 216856 \\
\hline \#11 & Cosmetic Techniques/ & 5700 \\
\hline \#12 & (cosmetic surgery or esthetic surgery).tw,kf. & 2371 \\
\hline \#13 & abdominoplasty/or lipoabdominoplasty/ & 763 \\
\hline \#14 & (abdominoplast* or lipoabdominoplast*).tw,kf. & 1910 \\
\hline$\# 15$ & Body Contouring/ & 269 \\
\hline \#16 & body contour*.tw,kf. & 1147 \\
\hline \#17 & Cervicoplasty/ & 172 \\
\hline \#18 & cervicoplast*.tw,kf. & 56 \\
\hline \#19 & $\begin{array}{l}\text { dermatologic surgical procedures/ or blepharoplasty/ or chemexfoliation/ or } \\
\text { dermabrasion/ or mohs surgery/ }\end{array}$ & 14731 \\
\hline \#20 & skin transplantation/ & 35862 \\
\hline \#21 & (skin adj3 (transplant* or graft*)).tw. & 22345 \\
\hline \#22 & $($ skin and (transplant* or graft*)).kf & 5354 \\
\hline \#23 & $\begin{array}{l}\text { (dermatoplast* or blepharoplas* or chemexfoliation or dermabrasion or mohs } \\
\text { surgery).tw,kf. }\end{array}$ & 4717 \\
\hline
\end{tabular}




\begin{tabular}{|c|c|c|}
\hline \#24 & Limb Salvage/ & 4359 \\
\hline \#25 & limb salvage.tw,kf. & 7271 \\
\hline \#26 & Lipectomy/ & 3682 \\
\hline \#27 & (lipectom* or lipoplast* or lipsuction or suction lipolysis).tw,kf. & 1166 \\
\hline \#28 & mammaplasty/ or breast implantation/ & 14375 \\
\hline \#29 & mamm\#plast*.tw,kf. & 3964 \\
\hline \#30 & (breast adj2 (implant* or reduc* or augment*)).tw. & 12196 \\
\hline \#31 & (breast and (implant* or reduc* or augment*)).kf. & 1661 \\
\hline \#32 & exp Mastectomy/ & 32631 \\
\hline \#33 & (mastectomy or breast resection or mammectomy).tw,kf. & 23853 \\
\hline \#34 & Rhinoplasty/ & 9765 \\
\hline \#35 & rhinoplast*.tw,kf. & 6163 \\
\hline \#36 & Sex Reassignment Surgery/ & 553 \\
\hline \#37 & (sex reassignment or gender confirmation).tw,kf. & 710 \\
\hline \#38 & tissue expansion/ or nerve expansion/ & 2383 \\
\hline \#39 & tissue expansion?.tw,kf. & 2256 \\
\hline \#40 & (nerve adj2 (elongation or expansion or lengthen* or stretch* or coaptation)).tw. & 660 \\
\hline \#41 & (nerve and (elongation or expansion or lengthen* or stretch* or coaptation)).kf. & 106 \\
\hline \#42 & Ulnar Collateral Ligament Reconstruction/ & 79 \\
\hline \#43 & ulnar collateral ligament reconstruction.tw,kf. & 231 \\
\hline \#44 & burns/ or burns, chemical/ or burns, electric/ & 53106 \\
\hline \#45 & burn*.tw,kf. & 106217 \\
\hline \#46 & Nerve Regeneration/ & 23210 \\
\hline \#47 & neurorrhaphy.tw,kf. & 704 \\
\hline \#48 & (nerve adj2 regenerat*).tw. & 8907 \\
\hline \#49 & (nerve and regenerat*).kf. & 2847 \\
\hline \#50 & Rhytidoplasty/ & 3483 \\
\hline \#51 & $\begin{array}{l}\text { (face lift? or platysmaplast* or platysmotom* or rhytidectom* or } \\
\text { rhytidoplast*).tw,kf. }\end{array}$ & 1896 \\
\hline
\end{tabular}




\begin{tabular}{|l|l|l|}
\hline$\# 52$ & Aesthetic surger*.tw,kf. & 1448 \\
\hline$\# 53$ & (breast adj2 (surger* or reconstruction)).tw. & 22997 \\
\hline$\# 54$ & (breast and (surger* or reconstruction)).kf. & 4994 \\
\hline$\# 55$ & (nerve adj3 (graft* or repair*)).tw. & 8526 \\
\hline$\# 56$ & (nerve and (graft* or repair*)).kf. & 871 \\
\hline$\# 57$ & Gynecomastia/ & 3434 \\
\hline$\# 58$ & Gynecomastia.tw,kf. & 3417 \\
\hline$\# 59$ & or/7-56 & 656463 \\
\hline$\# 60$ & 6 and 59 & 1683 \\
\hline$\# 61$ & exp animals/ not humans.sh. & 4805142 \\
\hline$\# 62$ & 60 not 61 & 1228 \\
\hline$\# 63$ & comment.pt. & 901126 \\
\hline$\# 64$ & 62 not 63 & 1200 \\
\hline
\end{tabular}

Cochrane Central Register of Controlled Trials (EBM Reviews). Search conducted in March, 2021.

\begin{tabular}{|l|l|l|}
\hline Search & Query & \multicolumn{1}{|c|}{$\begin{array}{c}\text { Records } \\
\text { retrieved }\end{array}$} \\
\hline$\# 1$ & Fibrin Tissue Adhesive/ & 499 \\
\hline$\# 2$ & ((fibrin or tissue) adj2 (glue or adhesive? or sealant? or spray)).tw. & 1337 \\
\hline$\# 3$ & ((fibrin or tissue) and (glue or adhesive? or sealant? or spray)).kw. & 136 \\
\hline$\# 4$ & (Artiss or Tisseel or Evicel or TachoSil or Evarrest or Raplixa).tw,kw. & 282 \\
\hline$\# 5$ & or/1-4 & 1565 \\
\hline$\# 6$ & Surgery, Plastic/ & 116 \\
\hline$\# 7$ & Reconstructive Surgical Procedures/ & 770 \\
\hline$\# 8$ & (plastic or reconstructive).tw,kw,jw. & 6403 \\
\hline$\# 9$ & reconstruction.tw,kw. & 8805 \\
\hline$\# 10$ & Cosmetic Techniques/ & 384 \\
\hline$\# 11$ & (cosmetic surgery or esthetic surgery).tw,kw. & 391 \\
\hline
\end{tabular}




\begin{tabular}{|c|c|c|}
\hline \#12 & abdominoplasty/ or lipoabdominoplasty/ & 35 \\
\hline \#13 & (abdominoplast* or lipoabdominoplast*).tw,kw. & 239 \\
\hline \#14 & Body Contouring/ & 7 \\
\hline \#15 & body contour*.tw,kw. & 91 \\
\hline \#16 & Cervicoplasty/ & 1 \\
\hline \#17 & cervicoplast*.tw,kw. & 0 \\
\hline \#18 & $\begin{array}{l}\text { dermatologic surgical procedures/ or blepharoplasty/ or chemexfoliation/ or } \\
\text { dermabrasion/ or mohs surgery/ }\end{array}$ & 511 \\
\hline \#19 & skin transplantation/ & 493 \\
\hline \#20 & $($ skin $\operatorname{adj} 3$ (transplant* or graft*)).tw. & 1277 \\
\hline \#21 & (skin and (transplant* or graft*)).kw. & 739 \\
\hline \#22 & $\begin{array}{l}\text { (dermatoplast* or blepharoplas* or chemexfoliation or dermabrasion or mohs } \\
\text { surgery).tw,kw. }\end{array}$ & 364 \\
\hline \#23 & Limb Salvage/ & 97 \\
\hline \#24 & limb salvage.tw,kw. & 344 \\
\hline \#25 & Lipectomy/ & 94 \\
\hline \#26 & (lipectom* or lipoplast* or lipsuction or suction lipolysis).tw,kw. & 47 \\
\hline \#27 & mammaplasty/ or breast implantation/ & 322 \\
\hline \#28 & mamm\#plast*.tw,kw. & 214 \\
\hline \#29 & (breast adj2 (implant* or reduc* or augment*)).tw. & 1720 \\
\hline \#30 & (breast and (implant* or reduc* or augment*)).kw. & 828 \\
\hline \#31 & exp Mastectomy/ & 1619 \\
\hline \#32 & (mastectomy or breast resection or mammectomy).tw,kw. & 4192 \\
\hline \#33 & Rhinoplasty/ & 262 \\
\hline \#34 & rhinoplast*.tw,kw. & 373 \\
\hline \#35 & Sex Reassignment Surgery/ & 2 \\
\hline \#36 & (sex reassignment or gender confirmation).tw,kw. & 8 \\
\hline \#37 & tissue expansion/ or nerve expansion/ & 40 \\
\hline \#38 & tissue expansion?.tw,kw. & 68 \\
\hline \#39 & (nerve adj2 (elongation or expansion or lengthen* or stretch* or coaptation)).tw. & 52 \\
\hline
\end{tabular}




\begin{tabular}{|c|c|c|}
\hline \#40 & (nerve and (elongation or expansion or lengthen* or stretch* or coaptation)).kw. & 55 \\
\hline \#41 & Ulnar Collateral Ligament Reconstruction/ & 1 \\
\hline \#42 & ulnar collateral ligament reconstruction.tw,kw. & 8 \\
\hline \#43 & burns/ or burns, chemical/ or burns, electric/ & 1496 \\
\hline \#44 & burn*.tw,kw. & 10237 \\
\hline \#45 & Nerve Regeneration/ & 73 \\
\hline \#46 & neurorrhaphy.tw,kw. & 19 \\
\hline \#47 & (nerve adj2 regenerat*).tw. & 156 \\
\hline \#48 & (nerve and regenerat*).kw. & 145 \\
\hline \#49 & Rhytidoplasty/ & 120 \\
\hline \#50 & $\begin{array}{l}\text { (face lift? or platysmaplast* or platysmotom* or rhytidectom* or } \\
\text { rhytidoplast*).tw,kw. }\end{array}$ & 73 \\
\hline \#51 & Aesthetic surger*.tw,kw. & 38 \\
\hline \#52 & (breast adj2 (surger* or reconstruction)).tw. & 4264 \\
\hline \#53 & (breast and (surger* or reconstruction)).kw. & 3761 \\
\hline \#54 & (nerve $\operatorname{adj} 3$ (graft* or repair*)).tw. & 234 \\
\hline \#55 & (nerve and (graft* or repair*)).kw. & 113 \\
\hline \#56 & Gynecomastia/ & 66 \\
\hline \#57 & Gynecomastia.tw,kw. & 289 \\
\hline \#58 & or/6-55 & 38379 \\
\hline \#59 & 5 and 58 & 244 \\
\hline
\end{tabular}

Cochrane Database of Systematic Reviews (EBM Reviews). Search conducted in March, 2021.

\begin{tabular}{|l|l|l|}
\hline Search & Query & \multicolumn{1}{|c|}{$\begin{array}{c}\text { Records } \\
\text { retrieved }\end{array}$} \\
\hline$\# 1$ & ((fibrin or tissue) adj2 (glue or adhesive? or sealant? or spray)).tw. & 85 \\
\hline$\# 2$ & ((fibrin or tissue) and (glue or adhesive? or sealant? or spray)).kw. & 13 \\
\hline$\# 3$ & (Artiss or Tisseel or Evicel or TachoSil or Evarrest or Raplixa).tw,kw. & 12 \\
\hline$\# 4$ & or/1-3 & 86 \\
\hline
\end{tabular}




\begin{tabular}{|c|c|c|}
\hline \#5 & (plastic or reconstructive).tw,kw,jw. & 409 \\
\hline \#6 & reconstruction.tw,kw. & 242 \\
\hline \#7 & (cosmetic surgery or esthetic surgery).tw,kw. & 7 \\
\hline \#8 & (abdominoplast* or lipoabdominoplast*).tw,kw. & 1 \\
\hline \#9 & body contour*.tw,kw. & 1 \\
\hline \#10 & cervicoplast*.tw,kw. & 0 \\
\hline \#11 & $($ skin $\operatorname{adj} 3$ (transplant* or graft*)).tw. & 75 \\
\hline \#12 & $($ skin and (transplant* or graft*)).kw. & 10 \\
\hline \#13 & $\begin{array}{l}\text { (dermatoplast* or blepharoplas* or chemexfoliation or dermabrasion or mohs } \\
\text { surgery).tw,kw. }\end{array}$ & 18 \\
\hline \#14 & limb salvage.tw,kw. & 31 \\
\hline \#15 & (lipectom* or lipoplast* or lipsuction or suction lipolysis).tw,kw. & 2 \\
\hline \#16 & mamm\#plast*.tw,kw. & 15 \\
\hline \#17 & (breast adj2 (implant* or reduc* or augment*)).tw. & 58 \\
\hline \#18 & (breast and (implant* or reduc* or augment*)).kw. & 2 \\
\hline \#19 & (mastectomy or breast resection or mammectomy).tw,kw. & 69 \\
\hline \#20 & rhinoplast*.tw,kw. & 4 \\
\hline \#21 & (sex reassignment or gender confirmation).tw,kw. & 1 \\
\hline \#22 & tissue expansion?.tw,kw. & 5 \\
\hline \#23 & $\begin{array}{l}\text { (nerve adj2 (elongation or expansion or lengthen* or stretch* or } \\
\text { coaptation)).tw. }\end{array}$ & 7 \\
\hline \#24 & (nerve and (elongation or expansion or lengthen* or stretch* or coaptation)).kw. & 0 \\
\hline \#25 & ulnar collateral ligament reconstruction.tw,kw. & 0 \\
\hline \#26 & burn*.tw,kw. & 1158 \\
\hline \#27 & neurorrhaphy.tw,kw. & 1 \\
\hline \#28 & (nerve adj2 regenerat*).tw. & 16 \\
\hline \#29 & (nerve and regenerat*).kw. & 0 \\
\hline \#30 & $\begin{array}{l}\text { (face lift? or platysmaplast* or platysmotom* or rhytidectom* or } \\
\text { rhytidoplast*).tw,kw. }\end{array}$ & 1 \\
\hline \#31 & Aesthetic surger*.tw,kw. & 6 \\
\hline
\end{tabular}




\begin{tabular}{|l|l|l|}
\hline$\# 32$ & (breast adj2 (surger* or reconstruction)).tw. & 94 \\
\hline$\# 33$ & (breast and (surger* or reconstruction)).kw. & 38 \\
\hline$\# 34$ & (nerve adj3 (graft* or repair*)).tw. & 13 \\
\hline$\# 35$ & (nerve and (graft* or repair*)).kw. & 1 \\
\hline$\# 36$ & Gynecomastia.tw,kw. & 10 \\
\hline$\# 37$ & or/5-36 & 1827 \\
\hline$\# 38$ & 4 and 37 & 35 \\
\hline
\end{tabular}

Embase. Search conducted in March, 2021.

\begin{tabular}{|c|c|c|}
\hline Search & Query & $\begin{array}{l}\text { Records } \\
\text { retrieved }\end{array}$ \\
\hline$\# 1$ & fibrin glue/ or platelet-rich fibrin/ & 11667 \\
\hline$\# 2$ & ((fibrin or tissue) adj2 (glue or adhesive? or sealant? or spray)).tw. & 11084 \\
\hline \#3 & ((fibrin or tissue) and (glue or adhesive? or sealant? or spray)).kw. & 2613 \\
\hline \#4 & (Artiss or Tisseel or Evicel or TachoSil or Evarrest or Raplixa).tw,kw. & 1483 \\
\hline \#5 & or $/ 1-4$ & 16401 \\
\hline \#6 & plastic surgery/ & 60853 \\
\hline \#7 & reconstructive surgery/ & 11700 \\
\hline \#8 & (plastic or reconstructive).tw,kw,jx. & 236466 \\
\hline \#9 & reconstruction.tw,kw. & 264793 \\
\hline$\# 10$ & esthetic surgery/ & 15193 \\
\hline \#11 & (cosmetic surgery or esthetic surgery).tw,kw. & 2834 \\
\hline$\# 12$ & abdominoplasty/ or lipoabdominoplasty/ & 2710 \\
\hline \#13 & (abdominoplast* or lipoabdominoplast*).tw,kw. & 2363 \\
\hline$\# 14$ & body contouring/ & 537 \\
\hline \#15 & body contour*.tw,kw. & 1947 \\
\hline \#16 & cervicoplasty/ & 121 \\
\hline \#17 & cervicoplast*.tw,kw. & 69 \\
\hline
\end{tabular}




\begin{tabular}{|c|c|c|}
\hline \#18 & skin surgery/ & 7102 \\
\hline \#19 & eyelid reconstruction/ & 6121 \\
\hline \#20 & chemexfoliation/ & 480 \\
\hline \#21 & skin abrasion/ & 3987 \\
\hline \#22 & mohs micrographic surgery/ & 1984 \\
\hline \#23 & skin transplantation/ & 13961 \\
\hline \#24 & $($ skin $\operatorname{adj} 3($ transplant* or graft*)).tw. & 26058 \\
\hline \#25 & $($ skin and (transplant* or graft*)).kw. & 2908 \\
\hline \#26 & $\begin{array}{l}\text { (dermatoplast* or blepharoplas* or chemexfoliation or dermabrasion or mohs } \\
\text { surgery).tw,kw. }\end{array}$ & 5721 \\
\hline \#27 & limb salvage/ & 9433 \\
\hline \#28 & limb salvage.tw,kw. & 9405 \\
\hline \#29 & lipectomy/ & 2301 \\
\hline \#30 & (lipectom* or lipoplast* or lipsuction or suction lipolysis).tw,kw. & 1390 \\
\hline \#31 & breast reconstruction/ or breast augmentation/ or breast reduction/ & 24581 \\
\hline \#32 & mamm\#plast*.tw,kw. & 4905 \\
\hline \#33 & (breast adj2 (implant* or reduc* or augment*)).tw. & 15065 \\
\hline \#34 & (breast and (implant* or reduc* or augment*)).kw. & 3175 \\
\hline \#35 & exp mastectomy/ & 61537 \\
\hline \#36 & (mastectomy or breast resection or mammectomy).tw,kw. & 35028 \\
\hline \#37 & nose reconstruction/ or nose septum reconstruction/ & 13515 \\
\hline \#38 & rhinoplast*.tw,kw. & 6134 \\
\hline \#39 & sex reassignment/ & 1233 \\
\hline \#40 & (sex reassignment or gender confirmation).tw,kw. & 1079 \\
\hline \#41 & tissue expansion/ & 3513 \\
\hline \#42 & nerve elongation/ or nerve reconstruction/ & 1803 \\
\hline \#43 & tissue expansion?.tw,kw. & 2769 \\
\hline \#44 & $\begin{array}{l}\text { (nerve adj2 (elongation or expansion or lengthen* or stretch* or } \\
\text { coaptation)).tw. }\end{array}$ & 829 \\
\hline \#45 & (nerve and (elongation or expansion or lengthen* or stretch* or coaptation)).kw. & 269 \\
\hline
\end{tabular}




\begin{tabular}{|c|c|c|}
\hline \#46 & ulnar collateral ligament reconstruction/ & 243 \\
\hline \#47 & ulnar collateral ligament reconstruction.tw,kw. & 274 \\
\hline \#48 & $\begin{array}{l}\text { burn/ or burn contracture/ or electric burn/ or esophagus burn/ or eye burn/ or } \\
\text { face burn/ or hand burn/ or lung burn/ }\end{array}$ & 64305 \\
\hline \#49 & burn*.tw,kw. & 132818 \\
\hline \#50 & nerve regeneration/ & 23718 \\
\hline \#51 & neurorrhaphy.tw,kw. & 790 \\
\hline \#52 & (nerve adj2 regenerat*).tw. & 10589 \\
\hline$\# 53$ & (nerve and regenerat*).kw. & 5985 \\
\hline \#54 & rhytidoplasty/ & 4204 \\
\hline \#55 & $\begin{array}{l}\text { (face lift? or platysmaplast* or platysmotom* or rhytidectom* or } \\
\text { rhytidoplast*).tw,kw. }\end{array}$ & 2136 \\
\hline \#56 & Aesthetic surger*.tw,kw. & 1774 \\
\hline \#57 & (breast adj2 (surger* or reconstruction)).tw. & 33224 \\
\hline \#58 & (breast and (surger* or reconstruction)).kw. & 7819 \\
\hline \#59 & (nerve adj3 (graft* or repair*)).tw. & 10030 \\
\hline \#60 & (nerve and (graft* or repair*)).kw. & 2194 \\
\hline \#61 & gynecomastia/ & 9628 \\
\hline \#62 & Gynecomastia.tw,kw. & 3918 \\
\hline \#63 & or/6-62 & 799184 \\
\hline \#64 & 5 and 63 & 2498 \\
\hline \#65 & $\begin{array}{l}\text { (exp animals/ or exp invertebrate/ or animal experiment/ or animal model/ or } \\
\text { animal tissue/ or animal cell/ or nonhuman/) and (human/ or normal human/ or } \\
\text { human cell/) }\end{array}$ & 22253358 \\
\hline \#66 & $\begin{array}{l}\text { (exp animals/ or exp invertebrate/ or animal experiment/ or animal model/ or } \\
\text { animal tissue/ or animal cell/ or nonhuman/) not } 65\end{array}$ & 6682100 \\
\hline \#67 & $64 \operatorname{not} 66$ & 1912 \\
\hline \#68 & editorial.pt. & 691056 \\
\hline \#69 & 67 not 68 & 1903 \\
\hline
\end{tabular}

Web of Science Core Collection. Search conducted in March 2021. 


\begin{tabular}{|c|c|c|}
\hline Search & Query & $\begin{array}{l}\text { Records } \\
\text { retrieved }\end{array}$ \\
\hline$\# 1$ & $\begin{array}{l}\text { TS=((fibrin NEAR/2 glue) OR (fibrin NEAR/2 adhesive\$) OR (fibrin } \\
\text { NEAR/2 sealant\$) OR (fibrin NEAR/2 spray) OR (tissue NEAR/2 glue) OR } \\
\text { (tissue NEAR/2 adhesive\$) OR (tissue NEAR/2 sealant } \$ \text { ) OR (tissue } \\
\text { NEAR/2 spray) OR (Artiss or Tisseel or Evicel or TachoSil or Evarrest or } \\
\text { Raplixa) ) }\end{array}$ & 11216 \\
\hline \#2 & $\mathrm{TS}=($ plastic or reconstructive $)$ & 373323 \\
\hline \#3 & $\mathrm{SO}=\left(\right.$ plastic $^{*}$ or reconstructive $\left.{ }^{*}\right)$ & 51257 \\
\hline \#4 & $\mathrm{TS}=($ cosmetic surgery or esthetic surgery $)$ & 16346 \\
\hline \#5 & TS=(abdominoplast* or lipoabdominoplast*) & 2204 \\
\hline \#6 & $\mathrm{TS}=($ body contour*) & 6838 \\
\hline \#7 & $\mathrm{TS}=($ cervicoplast $*)$ & 70 \\
\hline \#8 & $\mathrm{TS}=(($ skin NEAR/3 transplant* $)$ OR $($ skin NEAR/3 graft*) $)$ & 18382 \\
\hline \#9 & $\begin{array}{l}\mathrm{TS}=(\text { dermatoplast* } * \text { or blepharoplas* or chemexfoliation or dermabrasion } \\
\text { or mohs surgery) }\end{array}$ & 7894 \\
\hline$\# 10$ & $\mathrm{TS}=(\operatorname{limb}$ salvage $)$ & 8646 \\
\hline \#11 & $\mathrm{TS}=\left(\right.$ lipectom* ${ }^{*}$ lipoplast* or lipsuction or suction lipolysis) & 1492 \\
\hline$\# 12$ & $\mathrm{TS}=($ mamm?plast $*)$ & 4970 \\
\hline \#13 & $\mathrm{TS}=($ breast NEAR/2 (implant* or reduc* or augment*) $)$ & 16465 \\
\hline$\# 14$ & $\mathrm{TS}=($ mastectomy or breast resection or mammectomy) & 35450 \\
\hline \#15 & $\mathrm{TS}=($ sex reassignment or gender confirmation $)$ & 2698 \\
\hline \#16 & $\mathrm{TS}=($ rhinoplast $*)$ & 6,059 \\
\hline \#17 & $\mathrm{TS}=($ tissue expansion*) & 28160 \\
\hline \#18 & $\begin{array}{l}\mathrm{TS}=(\text { nerve NEAR/2 (elongation or expansion or lengthen* or stretch* or } \\
\text { coaptation) })\end{array}$ & 939 \\
\hline \#19 & TS=("ulnar collateral ligament reconstruction") & 209 \\
\hline$\# 20$ & $\mathrm{TS}=($ burns $*)$ & 34819 \\
\hline \#21 & $\mathrm{TS}=$ (neurorrhaphy) & 826 \\
\hline \#22 & $\mathrm{TS}=($ nerve $\mathrm{NEAR} / 2$ regenerat* $)$ & 14319 \\
\hline
\end{tabular}




\begin{tabular}{|l|l|l|}
\hline$\# 23$ & $\begin{array}{l}\text { TS=(face lift* or platysmaplast* or platysmotom* or rhytidectom* or } \\
\text { rhytidoplast*) }\end{array}$ & 4306 \\
\hline \#24 & TS=(Aesthetic surger*) & 10196 \\
\hline \#25 & TS=(breast NEAR/2 (surger* or reconstruction) $)$ & 28645 \\
\hline$\# 26$ & TS=(nerve NEAR/3 (graft* or repair*) $)$ & 9364 \\
\hline \#27 & TS=(Gynecomastia) & 2938 \\
\hline$\# 28$ & $\begin{array}{l}\text { \#27 OR \#26 OR \#25 OR \#24 OR \#23 OR \#22 OR \#21 OR \#20 OR } \\
\text { \#19 OR \#18 OR \#17 OR \#16 OR \#15 OR \#14 OR \#13 OR \#12 OR }\end{array}$ & 610131 \\
\#2 OR \#10 OR \#9 OR \#8 OR \#7 OR \#6 OR \#5 OR \#4 OR \#3 OR & \\
\hline \#29 & $\# 27$ AND \#1 & 1394 \\
\hline
\end{tabular}

LILACS. Search conducted in March, 2021.

\begin{tabular}{|l|l|l|}
\hline Search & Query & $\begin{array}{c}\text { Records } \\
\text { retrieved }\end{array}$ \\
\hline$\# 1$ & $\begin{array}{l}\text { Title, Abstract, Subject: ("Fibrin Tissue Adhesive") AND ("reconstructive" OR } \\
\text { "Plastic" OR "Cosmetic Techniques" OR "reconstruction") }\end{array}$ & 71 \\
\hline
\end{tabular}

Google Scholar. Search conducted in March, 2021.

\begin{tabular}{|l|l|l|}
\hline Search & Query & $\begin{array}{c}\text { Records } \\
\text { retrieved }\end{array}$ \\
\hline$\# 1$ & fibrin+"plastic|cosmetic|reconstructive AROUND(3) surgery" & 50 \\
\hline \multicolumn{2}{|l}{ Limited to articles from page 1 to 5 of 17,400 results. } \\
\hline
\end{tabular}

ProQuest Dissertations \& Theses Global. Search conducted in March, 2021.

\begin{tabular}{|l|l|c|}
\hline Search & Query & Records \\
& & retrieved \\
\hline
\end{tabular}




\begin{tabular}{|l|l|l|}
\hline$\# 1$ & $\begin{array}{l}\text { noft(fibrin) AND noft(((plastic NEAR/3 surgery) OR (cosmetic NEAR/3 } \\
\text { surgery) OR (resconstructive NEAR/3 surgery)) }\end{array}$ & 3 \\
\hline
\end{tabular}

OAlster. Search conducted in March, 2021.

\begin{tabular}{|l|l|l|}
\hline Search & Query & $\begin{array}{c}\text { Records } \\
\text { retrieved }\end{array}$ \\
\hline$\# 1$ & $\begin{array}{l}\text { ti:fibrin AND (kw:"plastic surgery" OR "cosmetic surgery" OR "reconstructive } \\
\text { surgery") }\end{array}$ & 13 \\
\hline \multicolumn{2}{|l}{ Limited to theses. }
\end{tabular}

World Health Organization (WHO) International Clinical Trials Registry Platform. Search conducted in March, 2021.

\begin{tabular}{|l|l|l|}
\hline Search & Query & $\begin{array}{c}\text { Records } \\
\text { retrieved }\end{array}$ \\
\hline$\# 1$ & $\begin{array}{l}\text { Condition: surgery } \\
\text { Intervention: fibrin }\end{array}$ & 12 \\
\hline
\end{tabular}

PapersFirst. Search conducted in March, 2021.

\begin{tabular}{|l|l|l|}
\hline Search & Query & $\begin{array}{c}\text { Records } \\
\text { retrieved }\end{array}$ \\
\hline$\# 1$ & $\begin{array}{l}\text { kw: fibrin and (kw: plastic w surgey OR kw: cosmetic w surgery OR kw: } \\
\text { reconstructive w surgery) }\end{array}$ & 3 \\
\hline
\end{tabular}

Proceedings. Search conducted in March, 2021.

\begin{tabular}{|l|l|l|}
\hline Search & Query & $\begin{array}{c}\text { Records } \\
\text { retrieved }\end{array}$ \\
\hline$\# 1$ & $\begin{array}{l}\text { kw: fibrin and (kw: plastic w surgey OR kw: cosmetic w surgery OR kw: } \\
\text { reconstructive w surgery) }\end{array}$ & 48 \\
\hline
\end{tabular}

